\title{
Using the Experimental Method to Calculate the Efficiency of Different Measurement Geometries for Reference Point Source Engineering using the NaI Detector (1) Detectors
}

\author{
Rawaa Yaseen Taha \\ Department of Physics, College of Science, Al-Mustansiriyah University, Baghdad, Iraq
}

\begin{abstract}
The quality of the gamma spectrometer results depends directly on the accuracy of the detection efficiency in the specific configuration of the concern. In this research, measurement conditions. In $\gamma$-ray spectroscopy using scintillation detectors, one usually needs to know the full-energy peak efficiency for any specific source where the detectors are calibrated for measuring point sources are placed in succession at constant distance from the considered detector to optimize the detection. Experimental calibration is limited to many measurement geometries and can not be directly applied to all measurement configurations. Hence, an alternative possibility of being able to calculate efficiency is highly desirable. The purpose of this research is calibration. The $\mathrm{Nal}$ ( $\mathrm{T} 1$ ) scintillation detector practically by using radioactive point sources in the development of axial dimensions from the surface of detector and with varying photon energies.
\end{abstract}

Key words: Gamma peak efficiency, efficiency calibration, full-energy peak efficiency, calibrated, efficiency, efficiency

\section{INTRODUCTION}

Measurements of the efficiency of point sources are usually performed and the corresponding calibration curve is created in a ray spectrometer either for the purpose of either subsequent measurement of the point sources of an unknown activity in the same geometry or to facilitate the efficient calculation of the expanded source. The use of point sources is a criterion in determining the Efficiency $\varepsilon(\mathrm{E})$ of the rays for either detectors (Radu et al., 2010). The experimental method is applied for the computation of the efficiency of the $\mathrm{NaI}$ (T1) scintillation detector for a point source located at several distances from the detector.

In this research, an analytical expression for the so-called full-energy peak Efficiency $\varepsilon(E)$ of the detector is defined as the quotient of the number of detected photons in a peak $\mathrm{N}_{\text {det }}$ and the total number of emitted photons in the same peak $\mathrm{N}_{\text {emit }}$ both per unit time interval. (Debertin and Helmer, 1988).

The Efficiency $(\varepsilon(\mathrm{E}))$ which is a dimensionless part is linked to a specific source, the detector geometry and a specific peak analysis (Haase et al., 1995).

\section{MATERIALS AND METHODS}

The calibration process was done by using (PTB) point sources, measured these sources placed in different geometries. In this research, different point sources are

\begin{tabular}{lccc}
\multicolumn{4}{l}{ Table 1: (PTB) point sources activities and their uncertainties } \\
$\begin{array}{lccc}\text { Uncertainty }(\%) & \begin{array}{c}\text { Reference } \\
\text { date }(12 \mathrm{H} 00)\end{array} & \text { Activity (kBq) } & \text { PTB nuclide } \\
(\mathrm{k}=2) & 1-6-2009 & 259.0 & { }^{241} \mathrm{Am} \\
2.6 & 1-6-2009 & 275.3 & { }^{133} \mathrm{Ba} \\
2.8 & 1-6-2009 & 385.0 & { }^{133} \mathrm{Cs} \\
4.0 & 1-6-2009 & 290.0 & { }^{152} \mathrm{Eu} \\
4.0 & 1-6-2009 & 212.1 & { }^{60} \mathrm{Co} \\
1.5 & \end{array}$
\end{tabular}

Table 2: Half-lives, photon energies and photon emission probabilities per decay for the all radionuclides used in this research

\begin{tabular}{lccc}
\hline Half life (days) Emission probability (\%) & Energy (keV) & Nuclide \\
\hline $1.58 \mathrm{E}+05$ & 35.30 & 0059.52 & ${ }^{241} \mathrm{Am}$ \\
$3.85 \mathrm{E}+03$ & 35.00 & 0080.99 & ${ }^{133} \mathrm{Ba}$ \\
$3.85 \mathrm{E}+03$ & 61.90 & 0356.01 & \\
$4.97 \mathrm{E}+03$ & 28.20 & 0121.78 & ${ }^{152} \mathrm{Eu}$ \\
$4.97 \mathrm{E}+03$ & 7.00 & 0244.70 & \\
$4.97 \mathrm{E}+03$ & 26.00 & 0344.27 & \\
$4.97 \mathrm{E}+03$ & 13.00 & 0778.90 & \\
$4.97 \mathrm{E}+03$ & 14.00 & 0964.00 & \\
$4.97 \mathrm{E}+03$ & 21.00 & 1407.90 & \\
$1.10 \mathrm{E}+04$ & 86.00 & 0661.64 & ${ }^{137} \mathrm{Cs}$ \\
$1.93 \mathrm{E}+03$ & 99.90 & 1173.23 & ${ }^{60} \mathrm{Co}$ \\
$1.93 \mathrm{E}+03$ & 99.98 & 1332.51 & \\
\hline
\end{tabular}

considered such as $\left({ }^{241} \mathrm{Am},{ }^{133} \mathrm{Ba},{ }^{137} \mathrm{Cs},{ }^{152} \mathrm{Eu}\right.$ and $\left.{ }^{60} \mathrm{Co}\right)$, all of the sources have the same geometric dimensions, reference date: 2009-06-01(12H00), these sources served as calibration solutions for systems used to measure full-energy peak efficiency at many distances from the detector. The certificates give the sources activities and their uncertainties for (PTB) sources are listed in Table 1. The data sheet states values of half lives, photon energies and photon emission probabilities per decay for the all radionuclides used in the calibration process are listed in Table 2. The axis of the sources was perpendicular to the 
Table 3: The Model 802 Scintillation detectors with the most common crystal sizes Well dimensions mm (inch)

\begin{tabular}{lcccr}
\hline L & D & Resolution & Crystal size (mm) (inch) & Model \\
\hline NA & NA & $7.5 \%$ & $76 \times 76(3 \times 3)$ & $802-3 \times 3$ \\
\hline
\end{tabular}

Table 4: The measuring times for different energy peaks along the vertical axis (the inclination angle $\theta=0^{\circ}$ )

\begin{tabular}{|c|c|c|c|c|c|c|c|c|}
\hline Height $(50 \mathrm{~cm})$ & Height $(45 \mathrm{~cm})$ & Height $(40 \mathrm{~cm})$ & Height $(35 \mathrm{~cm})$ & Height $(30 \mathrm{~cm})$ & Height $(25 \mathrm{~cm})$ & Height $(20 \mathrm{~cm})$ & & \\
\hline $\mathrm{T}_{\text {meas }}(\mathrm{sec})$ & $\mathrm{T}_{\text {meas }}(\mathrm{sec})$ & $\mathrm{T}_{\text {meas }}(\mathrm{sec})$ & $\mathrm{T}_{\text {meas }}(\mathrm{sec})$ & $\mathrm{T}_{\text {meas }}(\mathrm{sec})$ & $\mathrm{T}_{\text {meas }}(\mathrm{sec})$ & $\mathrm{T}_{\text {meas }}(\mathrm{sec})$ & Energy $(\mathrm{keV})$ & Nuclide IPL \\
\hline 2329.6 & 2748.7 & 2261.3 & 1818.1 & 1415.2 & 952.2 & 645.3 & 59.53 & ${ }^{241} \mathrm{Am}$ \\
\hline 1033.7 & 845.2 & 680.1 & 551.1 & 402.4 & 291.1 & 223 & 80.99 & ${ }^{133} \mathrm{Ba}$ \\
\hline 19219.2 & 14628.2 & 14481 & 10042.7 & 7870.5 & 5815 & 8721.3 & $\begin{array}{l}121.78 \\
244.7 \\
344.27 \\
778.9 \\
964 \\
1407.92\end{array}$ & ${ }^{152} \mathrm{Eu}$ \\
\hline 3559.9 & 1555 & 1258.4 & 987.5 & 821.8 & 619.7 & 432.1 & 661.66 & ${ }^{137} \mathrm{Cs}$ \\
\hline 5992.3 & 4952.5 & 3767 & 3069.7 & 2365.3 & 1691.3 & 1153 & $\begin{array}{l}1173.23 \\
1332.51\end{array}$ & ${ }^{60} \mathrm{Co}$ \\
\hline
\end{tabular}

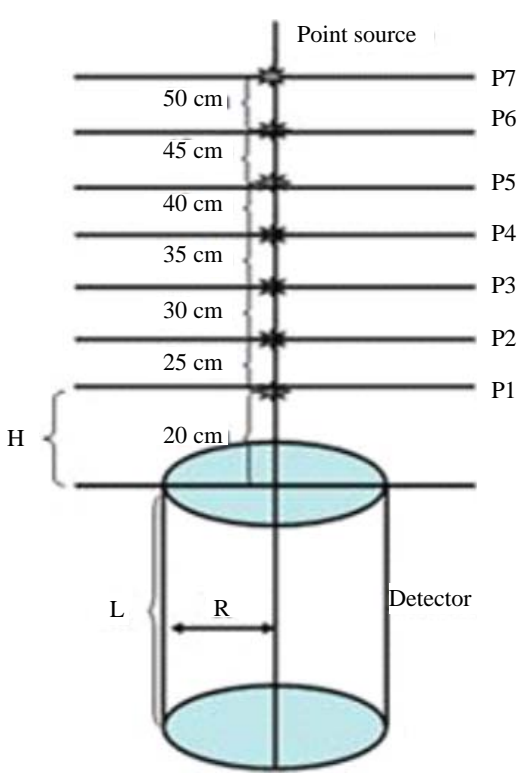

Fig. 1: Axial motion from the detector

detector axis. In order to keep the center of the sources on the detector axis, the sources were fixed on Plexiglas holders producing negligible attenuation (Aguiar et al., 2006).

All of the spectra for all point sources have been collected using scintillation detector with crystal size $(3 \times 3$ inch) with a high resolution $7.5 \%$. The details of the Model 802 Scintillation detector with the most common crystal sizes listed in Table 3 . Resolution is specified at the $662 \mathrm{keV}$ peak of ${ }^{137} \mathrm{Cs}$. Table 4 shows the measuring times for different energy peaks. The sources are placed at locations $20,25,30,35,40,45$ and $50 \mathrm{~cm}$ along the vertical axis plane, e.g. $\left(\theta=0^{\circ}\right)$ as shown in Fig. 1 .

\section{RESULTS AND DISCUSSION}

The full-energy peak efficiency values are measured as a function of the photon energy for The $\mathrm{NaI}$ (T1) scintillation detector determined by the following Eq. 1:

$$
\varepsilon(E)=\frac{N(E)}{T \cdot A_{s} \cdot P(E)} \Pi C_{i}
$$

Where:

$N(E)=$ The Number of counts in the full-energy peak which can be obtained using Genie 2000 Software

$\mathrm{T}=$ The measuring Time $(\mathrm{sec})$

$\mathrm{P}(\mathrm{E})=$ The photon emission Probability at Energy $(\mathrm{E})$

$\mathrm{A}_{\mathrm{S}} \quad=$ The radionuclide Activity

$\mathrm{C}_{\mathrm{i}}=$ The Correction factors due to dead time, radionuclide decay and coincidence summing corrections

The calibration source decay correction $C_{d}$ can be obtained from the decay constant $\lambda$ and the interval $\Delta \mathrm{T}$ between the reference time and the run time (Aguiar et al., 2006):

$$
\mathrm{C}_{\mathrm{d}}=\mathrm{e}^{\lambda \wedge \mathrm{T}}
$$

The statistical uncertainties of the net peak areas were smaller than $0.1 \%$, since, the acquisition time was long enough to get number of counts at least 10,000 counts. The uncertainty in the full-energy peak efficiency, $\sigma_{\varepsilon}$ was given by:

$$
\sigma_{\varepsilon}=\varepsilon \sqrt{\left(\frac{\partial \varepsilon}{\partial \mathrm{A}}\right)^{2} \cdot \sigma_{\mathrm{A}}^{2}+\left(\frac{\partial \varepsilon}{\partial \mathrm{P}}\right)^{2} \cdot \sigma_{\mathrm{P}}^{2}+\left(\frac{\partial \varepsilon}{\partial \mathrm{N}}\right)^{2} \cdot \sigma_{\mathrm{N}}^{2}}
$$



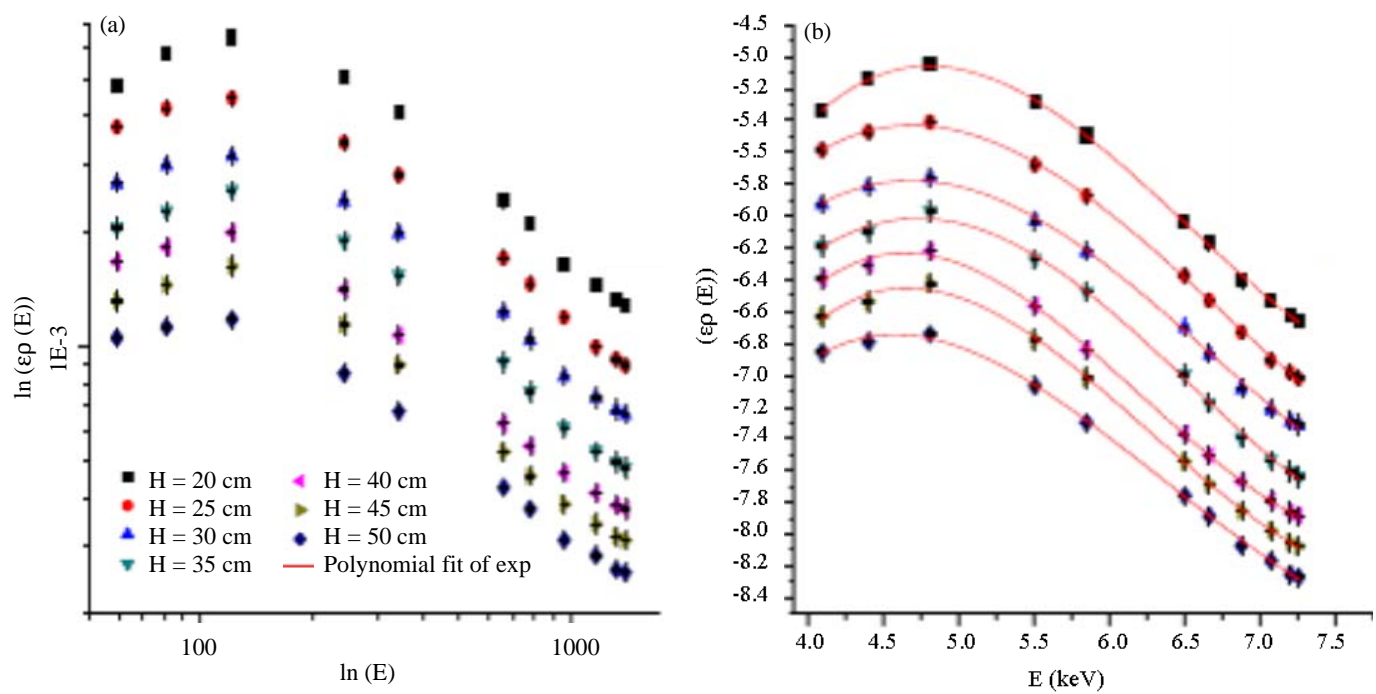

Fig. 2: a) The variation of efficiency with the energy at different heights from the detector center and b) Polynomial fit to the experimental efficiency with various gamma-ray energies

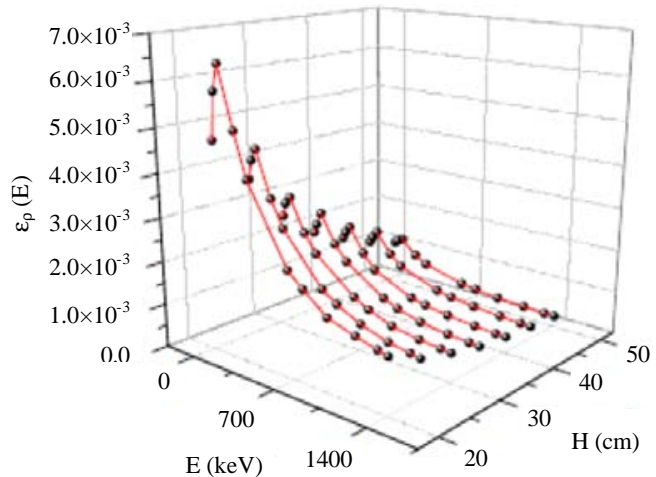

Fig. 3: Three dimensional presentation of the variation of efficiency as a function of energy at different heights as $\left(\theta=0^{\circ}\right)$

where, $\sigma_{A}, \sigma_{\mathrm{P}}$ and $\sigma_{\mathrm{N}}$ are the uncertainties associated with the quantities, $\mathrm{AS}, \mathrm{P}(\mathrm{E})$ and $\mathrm{N}(\mathrm{E})$, respectively, assuming that the only correction made is due to the source activity decay (Pibida et al., 2007; Cecil et al., 1985).

Figure 2 and 3 , the full energy peak efficiency $\left(\varepsilon_{\mathrm{p}}\right)$ increases gradually as a function of energy to reach a maximum at $\mathrm{E}=120 \mathrm{keV}$ and then decreases gradually to be very small at the high energy peak. Figure 4 the full-energy peak efficiency $\left(\varepsilon_{\mathrm{p}}\right)$ for low and medium energy $(59.52-661.64 \mathrm{keV})$ decreases remarkably as the height increases but in the high energy (778.9-1407.92 keV) the full-energy peak efficiency $\left(\varepsilon_{\mathrm{p}}\right)$ decreases slowly with increasing height, according to these data one an empirical formula (Fig. 5-7):

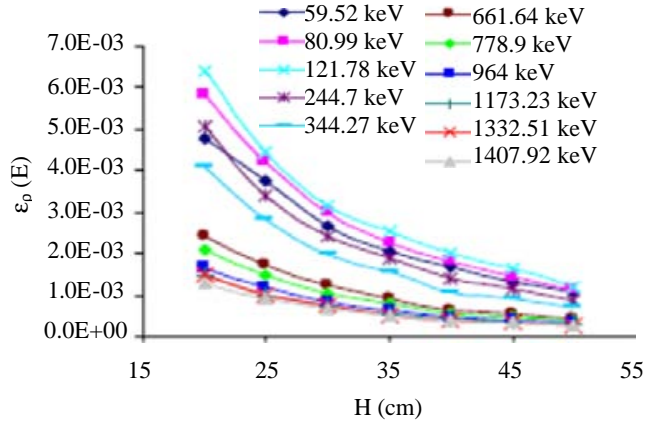

Fig. 4: The variation of efficiency with the height at $\left(\theta=0^{\circ}\right)$ for different energies

Table 5: Values of the constant (A) and (B) according to the source energy

\begin{tabular}{lcr}
\multicolumn{1}{c}{ and $\left(\theta=0^{\circ}\right)$} & & $\mathrm{E}(\mathrm{keV})$ \\
\hline $\mathrm{B}\left(\times 10^{-3}\right)$ & $\mathrm{A}\left(\times 10^{-3}\right)$ & 0059.53 \\
46.0 & 11.2 & 0080.99 \\
54.1 & 16.1 & 0121.78 \\
54.1 & 17.5 & 0244.70 \\
57.8 & 14.8 & 0344.27 \\
59.4 & 12.5 & 0661.66 \\
54.8 & 07.3 & 0778.90 \\
57.8 & 06.1 & 964.00 \\
55.8 & 04.7 & 1173.23 \\
54.3 & 03.9 & 1332.51 \\
57.84 & 04.2 & 1407.92 \\
53.5 & 03.4 &
\end{tabular}

$$
\varepsilon_{\mathrm{P}}=\mathrm{A}(\mathrm{E}) \mathrm{e}^{-\mathrm{B}(\mathrm{E}) \mathrm{H}}
$$

Where:

$\varepsilon_{\mathrm{p}} \quad=$ The experimentally measured efficiency,

$(\mathrm{A})$ and $(\mathrm{B})=$ Constants for a particular energy and certain angle $(\theta)$

$\mathrm{pH}=$ The height as show in Table 5 


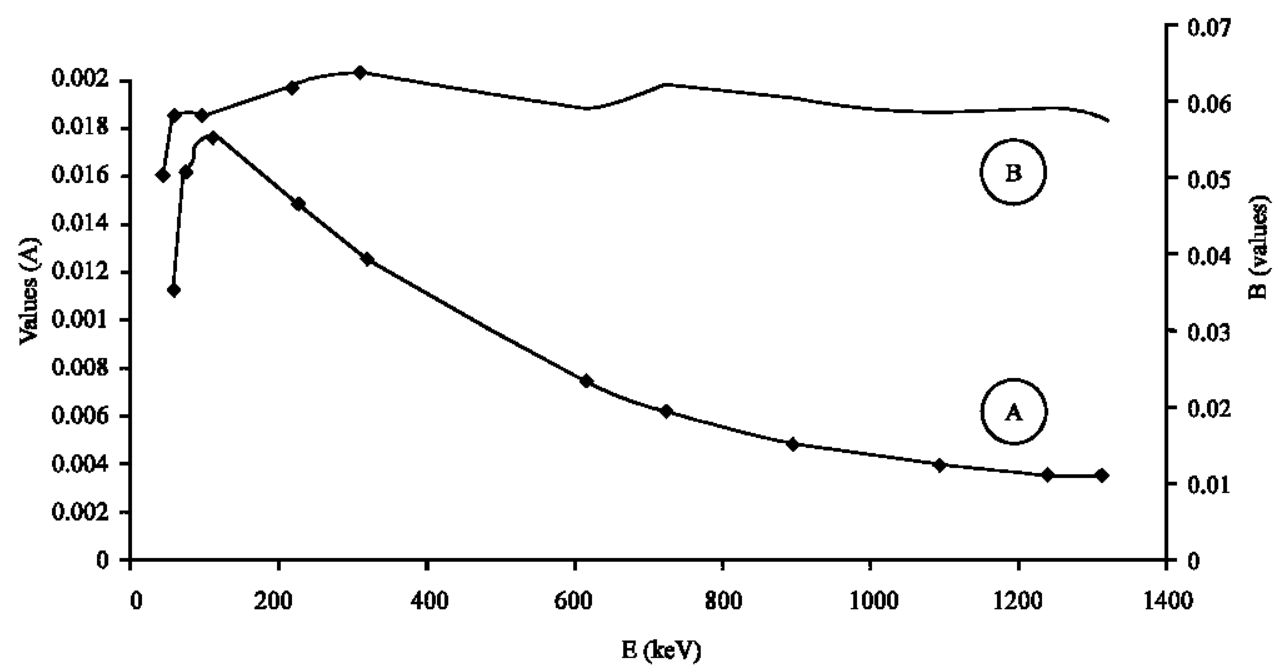

Fig. 5: The variation of constant values $(\mathrm{A}$ and $\mathrm{B})$ with the photon of energy at $\left(\theta=0^{\circ}\right)$ from the detector

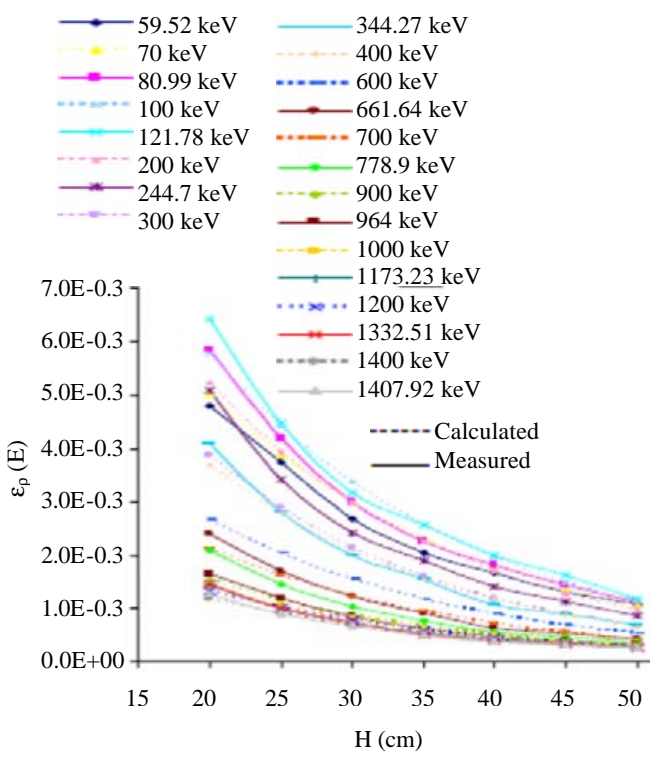

Fig. 6: The variation of measured and calculated efficiency with the height at $\left(\theta=0^{\circ}\right)$ from the detector

Figure 5 the values of the constant (A) is remarkably change with the energy of gamma photons while the constant $(\mathrm{B})$ is slightly changes with $(\mathrm{B})$. Where one are easily calculated the values of efficiency $\left(\varepsilon_{\mathrm{p}}\right)$ at any Height $(\mathrm{H})$ for a particular energy. The discrepancies between the calculated and the measured full-energy peak efficiency values are given by the following Eq. 5 :

$$
\mathrm{D} \%=\frac{\varepsilon_{\text {cal }}-\varepsilon_{\text {meas }}}{\varepsilon_{\text {meas }}} \times 100
$$

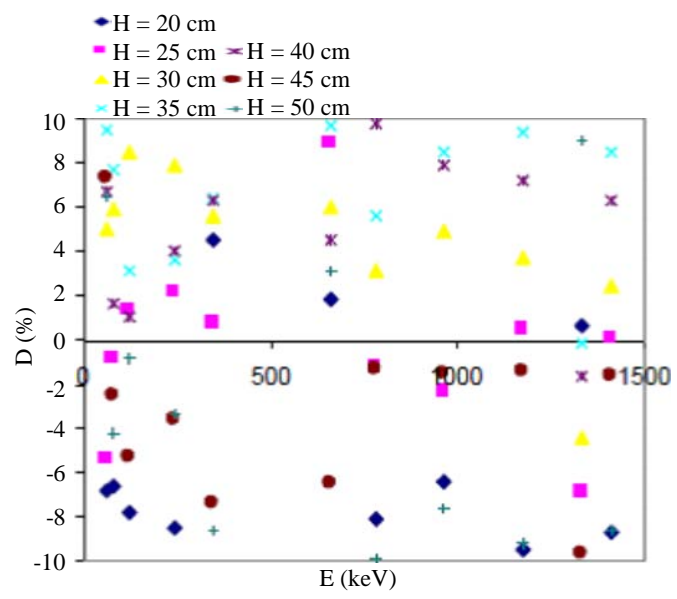

Fig. 7: The variation of deviation with the energy at different Heights $(\mathrm{H})$ along the vertical axis. The deviation shows a maximum at about $650 \mathrm{keV}$ and decreases to show a minimum at about $1300 \mathrm{keV}$

\section{CONCLUSION}

From the measurement of Efficiency $\varepsilon(\mathrm{E})$ and results that we obtained, we conclude the following; the full-energy peak efficiency is energy dependent and varying from energy region to another. At low energies, the full energy peak $\mu$ efficiency increases with energy to some point (about $81 \mathrm{keV}$ ) because in the dominant interaction in the detector material is the photoelectric effect. Whereas, in the energy range from $100-1000 \mathrm{keV}$ the Compton scattering takes place with high probability and some of the gamma rays are scattered and escape from the detector without detection, therefore, not all the 
photon energies contribute to the full-energy peak, so, the full-energy peak efficiency decreases gradually. At very high gamma ray energy $(\mathrm{E}>1.022 \mathrm{MeV})$, pair production is competing with Compton scattering when the probability of pair production increases with increasing energy, although the single and double escape peaks should be taken into account at high energy. In addition, the attenuation from the detector window, detector geometry and source-detector distance also is an issue and affect the efficiency $\left(\varepsilon_{\mathrm{p}}\right)$ of the detector as function of energy at the different height between the source and the detector along the vertical axis $\left(\theta=0^{\circ}\right)$ decreases exponentially by increasing height and photon energy. The best position of the point source to get high detector efficiency and high accuracy is at vertical height $(\mathrm{h}=20 \mathrm{~cm})$ where $\left(\theta=0^{\circ}\right)$. On the other hand, we found that the low detector efficiency at $(\mathrm{h}=50 \mathrm{~cm})$. Finally, this research can estimate $\mu$ efficiency for $\mathrm{NaI}$ (Tl) detector with crystal size ( $3 \times 3$ inch) with a high resolution $7.5 \%$ at any energy and any certain position.

\section{REFERENCES}

Aguiar, J.C., E. Galiano and J. Fernandez, 2006. Peak efficiency calibration for attenuation corrected cylindrical sources in gamma ray spectrometry by the use of a point source. Appl. Radiat. Isot., 64: 1643-1647.
Cecil, F.E., F.J. Wilkinson III, R.A. Ristinen and R. Rieppo, 1985. Experimental determination of absolute efficiency and energy resolution for $\mathrm{NaI}$ ( $\mathrm{Tl}$ ) and germanium gamma ray detectors at energies from 2.6 to $16.1 \mathrm{MeV}$. Nucl. Instrum. Methods Phys. Res. Sect. A. Accel. Spectrometers Detectors Associated Equip., 234: 479-482.

Debertin, K. and R.G. Helmer, 1988. Gamma-and X-ray Spectrometry with Semiconductor Detectors. 3rd Edn., North Holland Publishing, Amsterdam, Netherlands, ISBN-13:9780444871077, Pages: 399.

Haase, G., D. Tait and A. Wiechen, 1995. Determination of full energy peak efficiency for cylindrical volume sources by the use of a point source standard in gamma-spectrometry. Nucl. Instrum. Methods Phys. Res. Sect. A. Accel. Spectrometers Detectors Associated Equip., 361: 240-244.

Pibida, L., S.S. Nafee, M. Unterweger, M.M. Hammond, L. Karam and M.I. Abbas, 2007. Calibration of HPGe gamma-ray detectors for measurement of radioactive noble gas sources. Applied Radiat. Isot. J., 65: 225-233.

Radu, D., D. Stanga and O. Sima, 2010. Transfer of detector efficiency calibration from a point source to other geometries using ETNA software. Rom. Rep. Phys., 62: 57-64. 